\title{
Audience Behavior around Large Interactive Cylindrical Screens
}

\author{
Gilbert Beyer $^{1}$, Florian Alt ${ }^{2}$, Jörg Müller ${ }^{3}$, Albrecht Schmidt ${ }^{4}$, Karsten Isakovic ${ }^{5}$, \\ Stefan Klose $^{5}$, Manuel Schiewe ${ }^{5}$, Ivo Haulsen ${ }^{5}$ \\ ${ }^{1}$ Ludwig-Maximilians-University Munich, Germany, gilbert.beyer@ifi.lmu.de \\ ${ }^{2}$ University of Duisburg-Essen, Germany, florian.alt@uni-due.de \\ ${ }^{3}$ Deutsche Telekom Laboratories, TU Berlin, Germany, joerg.mueller@tu-berlin.de \\ ${ }^{4}$ VIS, University of Stuttgart, Germany, albrecht.schmidt@acm.org \\ ${ }^{5}$ Fraunhofer FIRST, Berlin, Germany, name.surname@first.fraunhofer.de
}

\begin{abstract}
Non-planar screens, such as columns, have been a popular means for displaying information for a long time. In contrast to traditional displays their digital counterparts are mainly flat and rectangular due to current technological constraints. However, we envision bendable displays to be available in the future, which will allow for creating new forms of displays with new properties. In this paper we explore cylindrical displays as a possible form of such novel public displays. We present a prototype and report on a user study, comparing the influence of the display shape on user behavior and user experience between flat and cylindrical displays. The results indicate that people move more in the vicinity of cylindrical displays and that there is no longer a default position when it comes to interaction. As a result, such displays are especially suitable to keep people in motion and to support gesture-like interaction.
\end{abstract}

\section{Author Keywords}

Cylindrical screens, digital columns, display formats, public displays, interactive surfaces, non-planar screens

\section{ACM Classification Keywords}

H.5.2. Information interfaces and presentation: User Interfaces.

\section{General Terms \\ Human Factors.}

\section{INTRODUCTION}

As display technology progresses and digital displays become cheaper, larger, and more robust, traditional displays in public spaces are being replaced by their digital counterparts. These digital displays can provide, among other bene-

Permission to make digital or hard copies of all or part of this work for personal or classroom use is granted without fee provided that copies are not made or distributed for profit or commercial advantage and that copies bear this notice and the full citation on the first page. To copy otherwise, or republish, to post on servers or to redistribute to lists, requires prior specific permission and/or a fee.

CHI 2011, May 7-12, 2011, Vancouver, BC, Canada

Copyright 2011 ACM 978-1-4503-0267-8/11/05...\$5.00. fits, interactivity, either by touch or by sensing the movement of the audience. Due to the deployed display technologies, nowadays the majority of displays are flat, rectangular, and framed. However, we learned from history, that there are many successful forms of non-planar displays.

One popular form were columns. Freestanding columns have the benefit of high visibility due to their concise and elevated shape, and can also provide more screen real estate on the same floor space. In addition, columns were extensively available inside buildings for structural reasons. Famous examples for ancient cylindrical displays are Trajan's Column in Rome (see Figure 1) or columns in the Hathor temple in Egypt. Even nowadays the most popular form of non-planar displays are cylindrical screens, such as cylindrical bulletin-boards, inflatable columns used at events, or street furniture columns used for cultural information, public announcements and ads (Morris or Litfaß Columns, see also Figure 1). With advances in technologies, bendable displays will allow for turning nearly any surface into a display and hence allow for creating displays of almost arbitrary shape and size for no additional costs compared to flat displays.
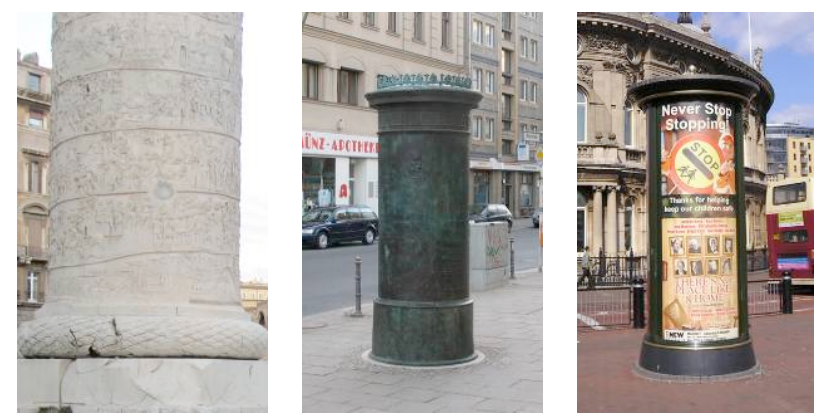

Figure 1: Examples of classical non-planar screens: (1) Trajan's Column with inscriptions in Rome (2) Replica of the first Litfaß Column in Berlin (3) Modern Citylight Column.

We opted to investigate cylindrical displays as one possible form of novel, arbitrary-shaped public displays. Hence we present a prototype of an interactive cylindrical display and 
report on a user study comparing user behavior in front of classical, flat displays and cylindrical displays showing the same content (we use the terms cylindrical display and column interchangeably). Our contribution is threefold:

- We present an interactive cylindrical display, which reacts to passers-by by adapting the content according to their movements.

- We show that people in front of cylindrical displays tend to move more and explore the content from a wider range of standing positions and discuss how this influences the way content should be designed.

- We discuss advantages and disadvantages of flat and cylindrical displays based on the findings of our study.

\section{RELATED WORK}

In recent years, a variety of prototypes of non-planar displays have been developed. Most public deployments, however, still use planar, framed displays and their designs are based on implicit assumptions that may not hold for nonplanar displays. While a number of studies of audience behavior for planar displays exist, their results may not generalize to non-planar displays. Finally, the specific case of cylindrical displays introduces the new category of semiframed displays.

\section{Interactive and Public Displays}

Inspired by early work such as Media Spaces [4] and the Digital Desk [29], a variety of interactive and public displays have been proposed. The Plasma Poster Network $[7,8]$ was a network of touch enabled public displays installed in hallways supporting office communication. Similar to our approach, many of such displays used computer vision to enable interaction from a distance. The seminal work of Myron Krueger explored vision-based interaction with large displays where the displays reacted to the users' movements [15]. In ReflectiveSigns [22], a network of displays reacted implicitly to the audience's viewing behavior. The Intelligent Kiosk [6] was an early example of an interactive Avatar on a public display that reacted to passing people. Similarly, the AmiQuin [26] was a virtual mannequin in a shop window that reacted to the audiences' body movements. Finally, Malik proposed vision-based interaction techniques with multiple fingers for distant displays [19].

\section{Designs of Non-Planar Screens}

Organic user interfaces [12] have been proposed as computer interfaces that use non-planar displays for input and output. Lin et al. presented the i-m-Tube, an interactive tubular display [18]. The first version uses a single projector and a convex mirror to create a back-projected cylindrical display, which supports multi-touch. Benko et al. present Sphere [1], a multi-touch enabled spherical display. They discuss unique properties of spherical displays and multitouch interaction techniques for such displays. Some of the properties for spheres are similar to properties of cylindrical displays (like that the user can not see the whole display at any time), while others are not (like a smooth transition between vertical and horizontal surfaces). Lee et al. [17] presented foldable displays like fans, newspapers, scrolls and umbrellas. Their prototypes were implemented using projection, infrared markers and the Nintendo Wii remote camera. They discuss affordances of different shapes and possible interaction techniques. Volumetric displays appear to show content inside the display volume. Grossmann et al. focus on such displays' unique properties and show how they can be used for collaborative applications $[9,10]$.

Regarding cylindrical displays, a number of commercial designs exist. Many commercial cylindrical displays use rotating LED's (Kinoton, Dynascan), where the drive system has to be well adjusted to get a jitter-free image. Other technical solutions include static mechanical designs including rings of LED modules (Barco) and projection-based setups. In [2], Benko outlines challenges when designing gestural interactions with non-flat surface computing interfaces derived from the development of three prototypes (a gesture-enabled flat display, a sphere, and a dome). Challenges identified include walk-up-and-use functionality, linking heterogeneous devices, usability from multiple directions, and compelling applications.

\section{Assumptions of Current Designs for Flat Displays}

Most current deployments of interactive public displays use planar, framed displays enabling interaction either through touch or body gestures. The CityWall [24], for example, was a large multi-touch display installed in downtown Helsinki that supported browsing photo collections. Worlds of Information [14] was an extension of the same system to include touch interaction with 3D spheres of photos. Magical Mirrors [21] was a deployment in downtown Berlin where passers-by could see their own mirror image on a display and interact with virtual objects through body movements. Such designs for flat displays usually start from a number of implicit assumptions that need to be questioned for non-flat displays. Many designs assume that: 1) people stop walking before they interact, 2) users can perceive the content of the entire screen at any time, 3) users can see what other people do when interacting with the display, 4) shoulders are usually parallel to the display, 5) the position centrally in front of the display is preferred, 6) content is not distorted. For example, touch displays are obviously difficult to use while walking, and even for Magical Mirrors a walking user would quickly leave the camera view. For the CityWall, users could easily scale photos so big that they hide the view for others, which would be a problem if users cannot observe the effects of their actions on other parts of the screen. Similarly, users started to play 'soccer' by throwing photos around, which would be very different if one could not observe the actions of other users. Vogel [28] used a parallel shoulder position as an indicator of user interest, but it is not clear if this is valid for nonplanar displays. Also, the distortion of content could render many current interfaces unusable on non-planar displays. 


\section{Audience Behavior in Front of Flat Displays}

Any observations of audience behavior towards public displays have been conducted using planar displays, and it is unclear whether existing findings generalize towards nonplanar displays. Scott et al. [27] explore how people use regions on round tabletops, which share some properties with cylindrical displays, but where the display itself is flat. Huang et al. [13] show that for existing planar displays, very few people look at them or stop in front of them. This may also be related to the fact that many flat displays are installed either on walls, orthogonal to walking direction, or above eye height. Cylindrical screens in contrast are often installed directly in the way of passers-by, such that they naturally appear in their field of view. Müller et al. [23] show that what people expect on public displays depends on the immediate surroundings, e.g. the apparent owner of a shop window where the display is installed. As columns are usually freestanding, expectations may differ. Brignull and Rogers [5] and Peltonen et al. [24] observed that users were waiting for their turn to access the display. For cylindrical displays, there is no central position where the user could 'own' the display, but all positions around the display are equal. Therefore, turn taking could be very different.

\section{Influence of the Display Frame on Audience Behavior}

A major difference between planar and non-planar screens may result from their frame. In this context Manovich [20] presents a theory of the imprisonment of the viewer's body by the screen apparatus on one hand and the requirements of the image perspective on the other hand. In cinema, e.g., the body of the viewer is confined to a seat and the head is aligned to forward view hence providing the best viewpoint. The same is true in classical arts (e.g., a painting in a museum) where the viewers seem to position themselves centrally at some distance in front of the screen. We show that this effect does not appear in front of non-planar screens. Another interesting conception of Manovich is the description of the screen's frame as a clearly defined rectangle, constituting a "viewing regime". Anything outside the frame can be ignored by the viewer, while immersing himself into the content inside the frame. Pinhanez and Podlaseck [25] discussed advantages and disadvantages of frameless displays, also claiming the significance of the frame to serve as a reference for the viewer to orient inside the scene and position himself accordingly. Cylindrical displays introduce a new category in between framed and frameless displays. It this case of semi-framed displays, a frame is provided on the top and bottom, but not left or right.

\section{PROTOTYPE OF A CYLINDRICAL DISPLAY}

For our research we developed a prototype of an interactive cylindrical display. Though our prototype is projector-based we envision that future versions will be based on bendable display foil. In the following, the hardware and software setup of this display is being described.

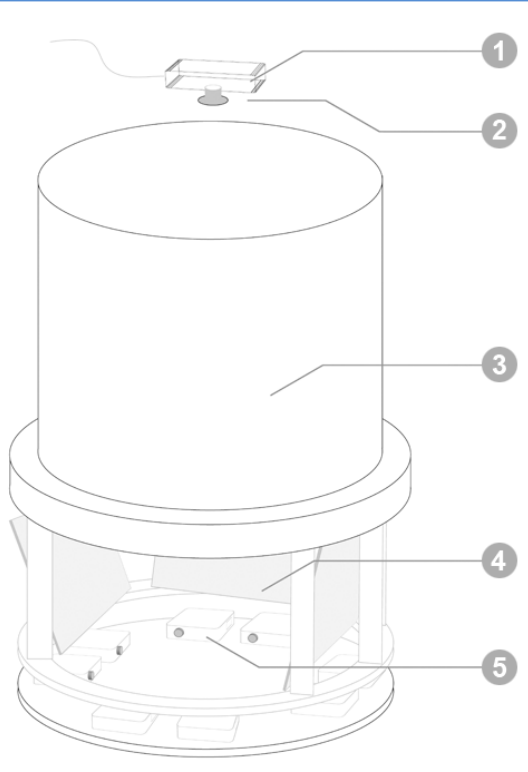

Figure 2: Prototype of a cylindrical display: (1) Camera sensor. (2) Fisheye lens. (3) Acrylic rear-projection screen. (4) $4 \times$ Foil mirror. (5) $8 \times$ Standard projector of $1024 \times 768$ pixels.

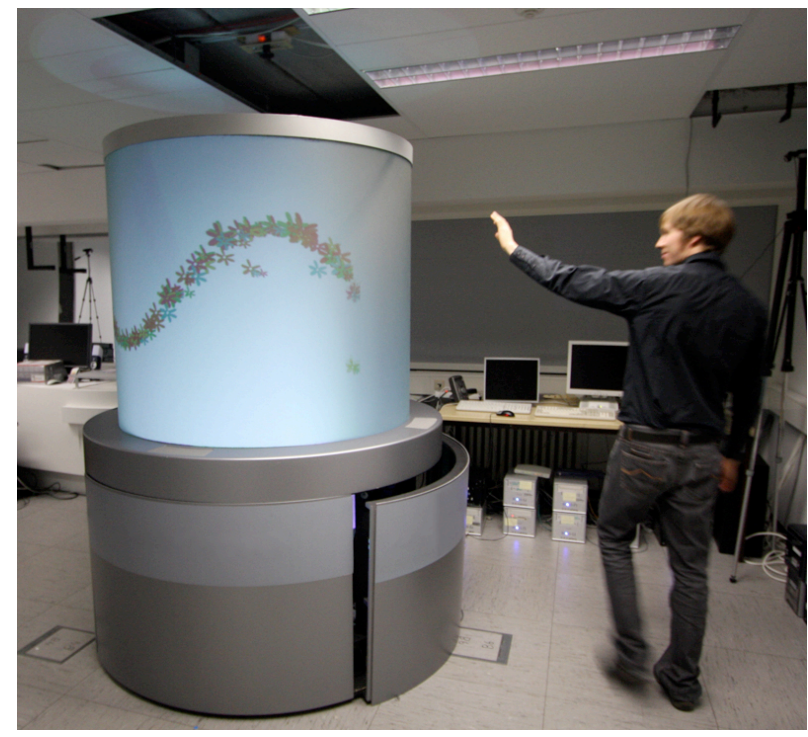

Figure 3: Cylindrical display with flower content for passing-by interaction as used in our user study.

\section{Hardware}

The prototype of the cylindrical display consists of a cluster of 8 standard projectors, 4 foil mirrors, 10 PCs, and a rearprojection screen. It has a height of 2.2 meters and a diameter of 1.5 meters. The $4: 1$ projection screen is 1.1 meters high, has a diameter of 1.3 meters and a resolution of 2048 to 512 pixels. Each cluster element projects onto a mirror reflecting the projection onto about one quarter of the screen. For a viewer independent image blending we use a special rear projection screen with a low gain factor. To de- 
tect position and movement of the passers-by around the column, a sensor interface is installed above the column consisting of a high-resolution camera and a $185^{\circ}$ fisheye lens. The hardware setup is depicted in Figure 2.

\section{Software}

The raw projection on the cylindrical screen is heavily distorted due to the curvature of the screen and the projection angle. To correct these distortions we use the calibration technology described in [3]. This software is also used to resolve the blending function for the real time correction between overlapping regions of adjacent projectors. The visualization software for the displayed contents on the column screen is based on a distributed rendering system. To enable user interaction, we implemented a motion tracking software, using OpenCV. The motion tracker uses frame differencing to detect motion, and calculates the angle, speed, and pixel distance of moving blobs from the column. The Kalman filter is used to smooth these trajectories.

We also developed a VRML-based application framework that enables us to display the same kinds of interactive applications within the coordinate systems of flat as well as round screen shapes. For the interactive digital column different sample applications were designed (see Figure 4):

1. Reactive animated typography. In this application words appear on the column as the user walks around it. While moving around the column it's in principle possible to explore content from left to right as well as from right to left. To make the text readable while passing clockwise as well as counterclockwise, both the horizontal and vertical dimensions are used. In order that words don't collide when taking a shift in direction, the text is presented on a visual step-curve. To ensure viewers do not lose track of the text flow, the next appearing word is always announced by an animated dot.

2. Direction of the viewer. In this application items are flying "towards" the user in $\mathrm{z}$ dimension, following the user by adjusting their horizontal direction. For example, the crown of a soft drink bottle seems to fly from the inside to the outside of the column and target the viewer, who cannot evade the item as he is tracked by the camera sensor. Such content, where the viewer cannot disregard the information, can be used for presenting urgent or provoking messages in social conscience campaigns for example.

3. Tales around the column. In this application, an endless picture story of drinking up a soft drink bottle is told around the column. With the help of the camera sensor the application presents the first picture on the side where the viewer is approaching, and animates the viewer to move further around the column and proceed in the story by providing information stepwise only. In order to see the end of the tale, the user has to circle the column.
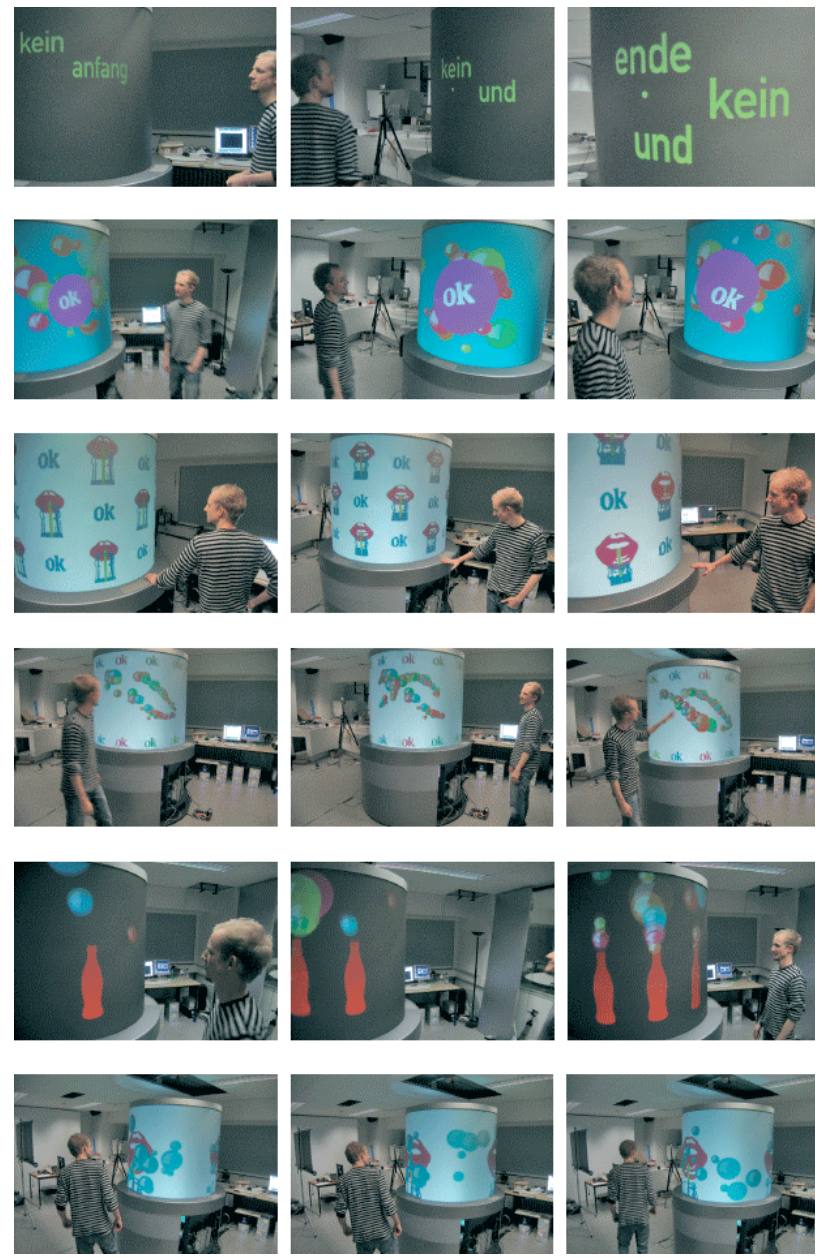

Figure 4: Developed applications for the cylindrical screen:

(1) Reactive animated typography. (2) Direction of the

viewer. (3) Tales around the column. (4) Movements of the viewer. (5) Reactive ambient column. (6) Interactive multiplayer game (detailed descriptions below).

4. Movements of the viewer. In this application, the user paints a pattern of flowers or bubbles on the column by any kind of body movement. If the user stops the flowers slowly fade away, and the individual pattern that has been painted can't be seen any more. Yet, when moving on the flowers reappear and follow the user as he moves along the surface, so that he is encouraged to proceed moving.

5. Reactive ambient column. In this application, each person approaching the column is represented by "being served" a soft drink bottle, which appears in the direction the person is standing. The aim is to give information about the public gathering and creating a social atmosphere. There have been reactive media façades in the past that present ambient information about the weather or about what is happening inside a building (e.g., a train station). The round nature of the column also allows mirroring objects in the outdoor space around the column, while a flat screen would only be able to cover one spatial direction. 
6. Interactive multiplayer game. This is a game where players are represented by individually colored bubbles on the screen appearing when moving fast, and have the task to burst all their opponents' bubbles. This game is making use of the fact that multiple users can interact with the round body of the column at the same time. In general all kinds of games are imaginable where teammates or opponents can chase each other around the column or hide behind it, like cops and robbers, hide and seek, etc.

Of these available applications, we chose application 4 for our study. This was because the application works for a single user and we were interested in user movement. The chosen application draws a flower pattern on the screen when the user moves, and he can influence the horizontal and vertical position of the flowers by walking left or right or by waving his hand. He can also influence the size of the flowers by moving faster or slower (see Figure 3 ).

\section{HYPOTHESES}

As no studies regarding user behavior towards cylindrical displays exist, first of all it is important to understand how people move and behave around them. This knowledge can then be used as a basis to develop applications that exploit the properties of the new format and investigate more elaborate topics such as multi-user interaction. As there are many (real-world) situations where only a single user is interacting, we decided to concentrate on single-user interaction only, leaving multi-user interaction for future work.

Based on informal observations of colleagues and visitors we posed three general hypotheses that characterize behavior around cylindrical displays. First, we assumed that users walk more when interacting with cylindrical than with flat displays. If true, this is an important property, as many flat displays are designed for people standing in front of them. Designs that work for people standing, with rather high complexity and small fonts, may not work for people walking. Second, we assumed that while users seem to have their shoulders parallel to flat displays, they would have their shoulders in a certain angle to columns. This would be an important property for gesture-based interaction, since while users can use both arms equally for flat displays, one arm would be turned away from a column, making symmetric gestures difficult. Further, it would be difficult to move any arm against the direction the user is facing, so a whole different gesture set would need to be designed. Finally, we hypothesized that due to the more active engagement users would spend more time interacting with columns.

\section{Hypothesis 1: Users walk more when interacting with} the cylindrical display.

1a: Users walk longer distances when interacting with the cylindrical display.

$1 \mathrm{~b}$ : Users spend more time walking when interacting with the cylindrical display. 1c: The position of users has a higher variance when interacting with the cylindrical display.

Hypothesis 2: Users position themselves with shoulders parallel to the flat display but not to the cylindrical display.

2a: The users' shoulder position is parallel to the display less often when interacting with the cylindrical display while walking.

$2 \mathrm{~b}$ : The users' shoulder position is parallel to the display less often when interacting with the cylindrical display while standing.

Hypothesis 3: Users spend more time overall interacting with the cylindrical display.

In addition, we had several hypotheses for the viewing behavior of participants. We hypothesized that participants would look more often at the cylindrical display, but for shorter bursts. We also hypothesized that participants would look at the left half of the column when walking clockwise and the right half when walking counterclockwise. While these hypotheses were formed before the design of the user study, we additionally conducted a post-hoc analysis of the data to explore further observations we made.

\section{USER STUDY DESIGN}

In order to test these hypotheses we conducted a user study comparing single users' behavior in front of interactive flat and cylindrical displays. The study was conducted at our lab over the course of two days. In the following chapter we describe the design and setup of our user study and report on the recruiting process as well as the study procedure.

We opted for a lab study due to the following reasons. (1) For the anticipated measurements, a highly controllable environment was required allowing for statistical data analysis. This would have been difficult to achieve in public due to a high amount of external influence and fragile, technical equipment. (2) To assess the users' behavior we used cameras during the study. This would have been a major issue in public due to privacy reasons. To create an authentic scenario we created a situation where participants were free to visit different rooms containing various exhibits. Hence we created a situation where (1) people behaved in a seminatural way, (2) people were not aware what we were measuring in order to avoid influencing their behavior, and (3) we created a controllable, yet still realistic scenario.

\section{Setup}

For the study we prepared 4 rooms at our lab each of which contained a prototype. Two of the rooms contained "fake" prototypes, which were functional (one was an interactive flat screen with content that reacted to the viewers' head movements and facial expressions, the other one was a noninteractive dome projection and showed a movie) - however their only purpose was to create a more realistic situation and to distract from the displays under investigation. 

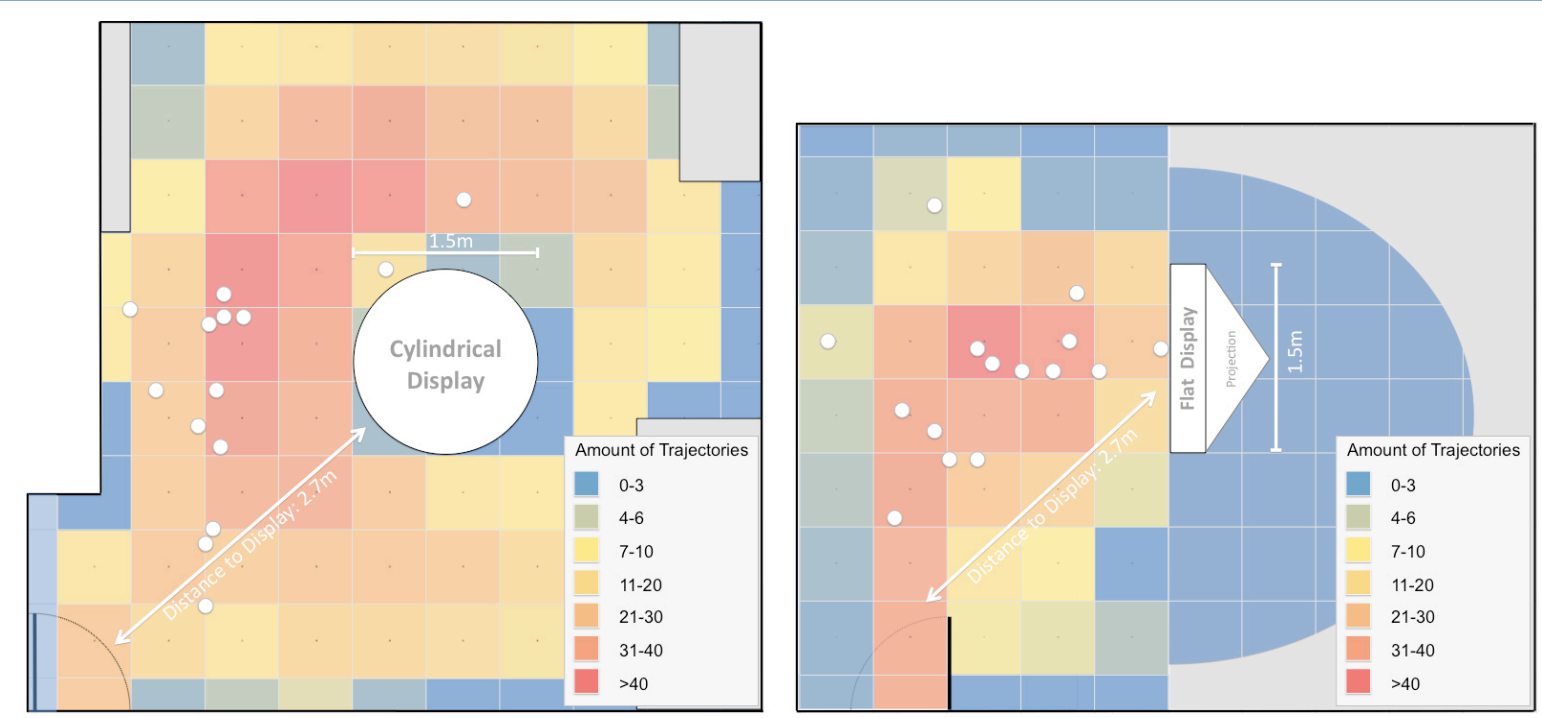

Figure 5: Room layout for the user study (1) Room with cylindrical display (2) Room with flat display. For the data analysis we separated the rooms into a grid consisting of $60 \times 60 \mathrm{~cm}^{2}$ squares. The heat map shows the participants' trajectories of each square. (White dots indicate participants' first standpoint after entering the room.)

For comparing the flat and the cylindrical display we designed two similar rooms, one containing the cylindrical display, and one the flat display (Figure 5). We designed the two rooms as similar as possible while still preserving the situation in which a flat or cylindrical display would normally be deployed. The distance between door and displays was equal and both displays would be approached in the same angle. While the doors would open in different directions, both displays would visually appear at the same position in the room. The flat display is equipped with one standard projector and uses the same rear-projection material as the cylindrical screen. For the size of the flat display we had to choose between a display that has the same size as the visible display area of the column when a user is standing in front of it, and the total size of the cylindrical screen 'unrolled'. Being in the situation to choose whether to deploy a flat or cylindrical display one would usually have to choose between two displays that take the same floor real estate and be in the first situation. For this practical reason, we decided to test a flat display with the same size as the visible area of the column first and leave the second situation for future work. The flat display uses a webcam as a sensor. For user tracking we setup 4 webcams in each room, which allowed for detecting the user in each position of the room. From the webcams we created a synchronized, time-stamped video file using OpenCV. To be able to analyze and manually annotate the video files we attached markers to the floor hence creating a grid of $60 \times 60$ $\mathrm{cm}^{2}$ squares.

\section{Participants}

We aimed for diverse participants as would normally be found in public spaces. People were recruited from bulletins in the surrounding neighborhoods (train stations, shopping malls, university campus, etc.) and mailing lists in the days prior to the study. In total, 15 people participated in the study (10 males, 5 females). The average age was 32.7 years; participants were students, artists, taxi drivers, office workers, and technicians.

\section{Procedure}

The participants were invited to the labs and asked to report to the doorman from where we led them to the laboratory. We started with an initial briefing. First, we had the participants fill in a demographic questionnaire (asking for age, gender, nationality, profession, and, if applicable, their major). Second, we explained them the setting of the study. They were told that there were 4 exhibition rooms containing different project works. We did not tell them which or how many expositions were to be found in each room. Instead, we asked them to simply walk through the rooms and spend as much time there as they wanted. We especially told them that there was no minimum and no maximum time we expected them to spend in each room, in order to minimize compliance effects. We told them that a one-page questionnaire was to be found after each room and asked them to fill it in once they finished their visit to the room. After that we wanted them to move on to the next room. Further we told them that several cameras were setup in the rooms, needed for the purpose of the study (we did not tell the participants that some prototypes were interactive).

After the initial briefing we guided the participants to the first room and explained them that arrows attached to the walls and floors would lead the way through and to further rooms. Though we surveyed the people in the different rooms (using webcams), we did not interrupt or talk to them during the study. Since we used a within-subject design (all participants saw all expositions) the order of the exhibits 
was counter-balanced among the subjects. Whereas the "fake" prototypes were always seen in the same order, we switched positions for the rooms containing the flat/cylindrical screens. After the participants finished the fourth questionnaire, we conducted a semi-structured interview with them. All semi-structured interviews were audiorecorded. Finally we debriefed the participants and explained them the purpose of the study. Participants were compensated with a small amount of money.

\section{DATA ANALYSIS}

Complete time-stamped and synchronized videos from four different perspectives of all user behavior were recorded in both rooms. All user positions were transcribed. A coding schema was devised containing codes for walking, shoulder angle, and head direction. The complete video recordings were coded by two independent raters using Mangold INTERACT coding software. In order to ensure inter-rater reliability, one video was coded by both raters and Cohen's Kappa was computed for all codes. Inter-rater reliability was satisfactory for walking $(\mathrm{Kappa}=.61)$ and shoulder $(\mathrm{Kappa}=.67)$ codes (we considered Kappa $\geq .61$ substantial agreement [16]). Unfortunately, reliability was not satisfactory for head direction codes, such that we had to drop all hypotheses related to viewing behavior. In addition to the testing of the hypotheses, a post-hoc analysis of various variables of user behavior (e.g., duration of stops) was conducted. Because we could not ensure normal distribution for all dependent variables, all hypotheses were tested using the non-parametric Wilcoxon signed-rank test with paired samples using a level of significance of .05. The AttrakDiff questionnaire was evaluated using the associated software, and semi-structured interviews were partially transcribed to cover non-repetitive user statements.

\section{RESULTS}

In the following, we report on the results of our data analysis. An overview of the results is presented in Table 1.

\section{Walking}

All hypotheses regarding the walking behavior of participants (hypotheses 1a, 1b, and 1c) are supported by the data. Participants covered significantly more meters with the column screen (on average $47.3 \mathrm{~m}$ ) than with the flat screen $(21.2 \mathrm{~m})(\mathrm{p}<.003)$ (see Figure 9). With the column, participants spent most of their time walking (only $44.9 \%$ standing), while with the flat display, they spent most of their time standing $(62.8 \%)(\mathrm{p}<.027)$. Also, they covered many more different locations with the column. The variance of location in rows is more than twice as high (5.6) for the column than for the flat screen $(2.3)(\mathrm{p}<.001)$, and the variance in columns is more than three times as high (3.7) for the column than for the flat screen $(.93)(\mathrm{p}<.001)$.

\section{Shoulders}

The hypotheses of the angle of shoulders towards the display are partially supported. Overall, participants spent most of their time with their shoulders parallel to the flat display (69.5\%) (see Figures 7 and 8).

\begin{tabular}{|c|c|c|}
\hline Measure & $\begin{array}{l}\text { Column } \\
(\text { mean } / \text { std })\end{array}$ & $\begin{array}{c}\text { Flat } \\
(\mathrm{mean} / \mathrm{std})\end{array}$ \\
\hline Distance walked $(\mathrm{m})^{* *}$ & $47.3 / 24$ & $21.2 / 13.7$ \\
\hline Time spent standing $(\%)^{*}$ & $44.9 / 25.9$ & 62.8/16.6 \\
\hline Mean duration of stops $(\mathrm{s})^{* *}$ & $3.5 / 2.3$ & 9.9/13.1 \\
\hline Max duration of stops (s)* & $12.9 / 11.9$ & $38.7 / 52.7$ \\
\hline Total time spent $(\mathrm{s})^{* *}$ & $97.7 / 53.4$ & 172.8/138.6 \\
\hline $\begin{array}{l}\text { Time spent with shoulders par- } \\
\text { allel }(\%)^{* * *}\end{array}$ & $41.5 / 21.3$ & 69.5/17.2 \\
\hline $\begin{array}{l}\text { Time spent with shoulders par- } \\
\text { allel while walking }(\%)^{* * *}\end{array}$ & $22.1 / 10.6$ & 46.3/16.1 \\
\hline $\begin{array}{l}\text { Time spent with shoulders par- } \\
\text { allel while standing }(\%)\end{array}$ & $70.0 / 26.1$ & $82.0 / 18.6$ \\
\hline Stops per minute $(1 / \mathrm{min})$ & $8.3 / 4.3$ & $6.8 / 3.3$ \\
\hline Mean distance from display (m) & $1.5 / .23$ & $1.7 / .41$ \\
\hline Variance in location (Rows) $* * *$ & $5.6 / 1.4$ & $2.3 / .96$ \\
\hline $\begin{array}{l}\text { Variance in location (Col- } \\
\text { umns)*** }\end{array}$ & $3.7 / 1.4$ & $.93 / .57$ \\
\hline
\end{tabular}

Table 1: All comparisons with Wilcoxon signed-rank test with paired samples $(*=p<.05, * *: p<.01, * * *: p<.001)$
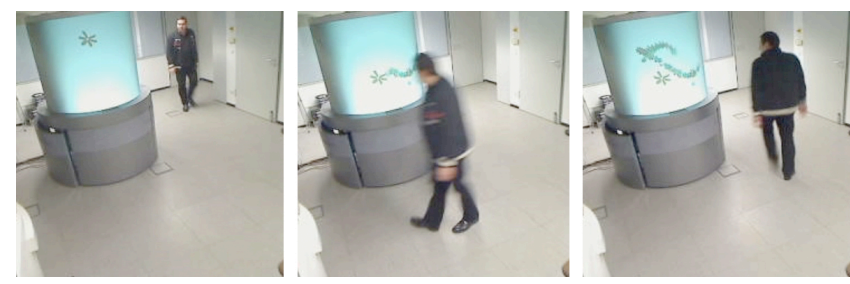

Figure 6: Typical user behavior in front of cylindrical display (1) User gets engaged into interaction immediately. (2) User keeps waking around the display. (3) Shoulder position is mainly orthogonal throughout interaction phase.
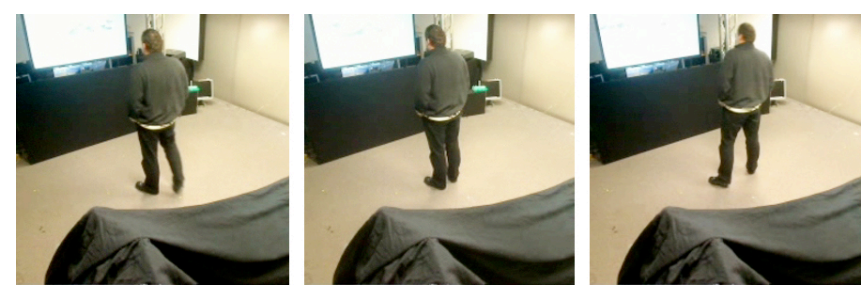

Figure 7: Typical user behavior in front of flat display (1) User approaches display (2) User stops in front of display. (3) User maintains a parallel shoulder position, even during interaction.

In contrast, with the column participants spent most of their time with shoulders not parallel (only $41.5 \%$ parallel) $(p<.001)$ (see Figures 6 and 8). If this data is divided by whether participants were walking or not, the picture is more diverse. When walking, participants spent $46 \%$ of their time shoulders parallel to the flat display, while this was the case for the column only $22 \%$ of the time $(p<.001)$. 
When standing, there is a trend towards participants having their shoulders parallel to the flat display more often $(81 \%)$ than to the column (69\%), however, this difference is not significant $(\mathrm{p}<.069)$. While hypothesis $2 \mathrm{a}$ is supported by the data, hypothesis $2 \mathrm{~b}$ is not.

\section{Time Spent for Interaction}

Hypothesis 3 assumed that participants would spend more time with the column than with the flat display. This hypothesis is not at all supported by the data. Indeed, participants spent almost twice as much time with the flat display (2:53 min) than with the column $(1: 38 \mathrm{~min})$. A post-hoc analysis shows that this is significant $(\mathrm{p}<.002)$ (Figure 9).

\section{Post-hoc Analysis}

When participants stopped for the first time in front of the flat display, most participants stopped in a very narrow area in front of it, while the locations of first stops were more diverse for the column (see Figure 5). The same figure also shows a heat map of where participants moved. This also nicely shows the "sweet spot" area in front of the flat display, which is much more spread out for the column. Also, participants spent slightly more time until they first stopped with the column $(10.4 \mathrm{~s})$ than with the flat display $(6.7 \mathrm{~s})$, and then continued walking sooner (after 5.7s) than with the flat display (22.5s). These differences however are not significant. Participants stopped slightly more often (per minute) with the column (8.3 stops) than with the flat display (6.8 stops), although this difference was not significant. However, if participants stopped, they stopped for more than twice as long $(9.9 \mathrm{~s})$ with the flat display than with the column $(3.5 \mathrm{~s})$, and this difference is significant $(\mathrm{p}<.006)$. The longest time participants spent without moving is even more extreme. While with the column, participants spent on average no more than $12.9 \mathrm{~s}$ on a single location, with the flat display, participants spent up to $38.7 \mathrm{~s}$ in a single location. Additionally, participants seemed to approach the column a little bit closer (avg. distance $1.5 \mathrm{~m}$ ) than the flat display $(1.7 \mathrm{~m})$, however, these differences are not significant.

\section{User Experience}

To evaluate the user experience we had the participant fill in an AttrakDiff questionnaire [11] for both the flat and the cylindrical display. The results revealed that from a pragmatic as well as from a hedonic point of view the participants considered the cylindrical display to be of higher quality. Considering the attractiveness, the cylindrical display was rated very attractive whereas the flat display was rated medium attractive. All results from the questionnaire were not significant, which might have been caused by our rather small sample size.

\section{Semi-Structured Interviews}

The participants did not seem to feel bothered by the experimental setup: "As soon as you start to play around with the media, you forget that you are observed." (P2) Interestingly, many participants experienced the flat display like 'television' (e. g. P3, P4, P7), while they experienced the column as being more 'dynamic': "The flat was like a television feeling. The round was more dynamic. TV is more static." (P3) They also felt more passive in front of the flat display: "You stand in front of it like in front of a TV.

While with the column you are engaged more actively. It was more like a TV situation; you walk in front of it and think, what happens now. With the column, you immediately had the feeling, you do more with it." (P7) Some participants considered the column as more comfortable: "It was more spatial. [...] It is better if you can move around a fixed point than back and forth." (P1) "For me, round is more comfortable than square. It was more organic.” (P3)

\section{DISCUSSION}

Our study revealed that single user behavior differs significantly between planar and cylindrical displays. Due to the setup of the study, there are certain limitations with regards to the generalizability of the results. (1) We only evaluated single user interaction. Hence no conclusion can be drawn towards behavior in multi-user scenarios. (2) Unlike in the lab, for real world deployments there are many factors influencing movement patterns, such as streets, buildings, or pedestrian traffic. However, as columns are often deployed in small free places, spatially similar to our lab setting, we believe that the observed movement patterns generalize to such situations. (3) With regard to the content, we believe that the findings are valid for most similar interactive content. Yet, very different content (e.g., a fixed framed video), unsuitable for the column, might lead to different results.

In the following we outline and discuss major findings.

\section{Moving Around the Column}

As opposed to a flat, rectangular display, a column does not provide any boundaries left or right. This lack of borders indeed seems to have the effect of making viewers move freely around the column (see Figures 5 and 6). Participants spent most of their time walking and covered significant distances, looking at the column from various locations and stopping quite often, but only for relatively short times, on average only 3.5 seconds. This led to much more diverse body postures as opposed to a flat display. Only about $30 \%$ of the time was spent with shoulders parallel to the display, and even when participants stopped, $30 \%$ of the time the shoulders were not parallel.

\section{The Sweet Spot}

The data shows that for flat displays, there is a relatively small area in front of the display where participants get themselves in a frontal position ('the sweet spot'). Though this looks like an obvious finding, we are not aware of any prior studies that support this assumption with data. This area was positioned centrally in front of the display, about 1.5 meters away from it. Participants seemed to approach this area quickly after entering the room, and stopped in this position with their shoulders parallel to the display, facing the display frontally (see Figures 5 and 7). From this position, they could see the entire screen from the best perspec- 
tive, while the entire frame was still in the visual field. These observations are well aligned with the theory of Manovich [20]. Participants stopped in the same position for quite long times, on average for up to 39 seconds, with one participant not moving for more than three minutes. When they moved, almost half of the time they still had their shoulders parallel to the display, moving back- and forwards, or sideways without turning. Overall they spent most of their time standing and did not cover great distances.

\section{Time Spent for Interaction}

As opposed to the hypothesis, participants spent significantly more time with the flat display than with the column, almost twice as long. This is an interesting finding, which we believe is worthwhile to be investigated in more detail. As people stand still to identify with the screen image and ignore the real world, they might easily spend more time than if moving around, exploring also the physical space.

\section{RECOMMENDATIONS}

Based on these results we derive recommendations for designing content on planar and cylindrical displays.

\section{Design for Walking Interaction}

Cylindrical displays are most suitable to keep people in motion, even if they do not frontally face the display. Unlike flat displays where motion is often simulated in an unnatural way (e.g., "running" in the same spot in front of a flat display using Xbox Kinect Sports track-and-field), cylindrical displays provide a way to support real motion. Thus, columns and their content should be designed for walking, and be set up at sites where they can raise the attention of viewers by implicit interaction and encourage them to move on. A way to achieve this is to keep content simple, such that it can be perceived while passing by. Another good way is content that moves with the audience as they move around the column, adapting to the location of the viewer rather than requiring the viewer to adapt his location to the display. We propose that flat displays, because of their sweet spot, may be more suited for waiting situations and longer dwell times, and may support more complex content.

\section{Place Columns in the Way of Users}

Cylindrical displays are suitable to be located within the way of users and can better be used while passing by. For flat displays people tend to take a fixed standing position for interacting, probably blocking the way for others. In contrast, cylindrical displays allow users to avoid bumping into other people by keeping moving around the display. As a result, content on cylindrical displays should adapt to users passing by and could, e.g., attract them towards noncrowded areas around the display.

\section{Enable Gesture-Based Interaction}

Previous research showed that three interaction techniques are highly suitable for the use on public displays: touch, gestures/body position, and interaction through mobile phones. Whereas touch and mobile phone based interaction is applicable for flat displays where people stop in front, the motion-fostering character of cylindrical displays makes them more suitable for gesture-like interaction. We observed that almost $60 \%$ of the time, the viewers' shoulders were not parallel to the display, leading to that they could not use both hands equally well for interaction as one arm is constantly turned away from the display. It may be best to enable interaction with one arm alone, and not requiring movement of the arm against walking direction. As another difficulty, which arm is geared towards the display depends on walking direction and not on dominance of the hand.

\section{Use Frameless or Semi-Framed Content}

Digital columns are semi-framed. As a result they don't have a "sweet spot" any more, hence posing another difficulty since designers and audience can't use left or right frames to orient themselves anymore. Using framed content, like videos or images, on a column, of course can create a virtual frame (and thus probably another 'sweet spot'), but that may not put the cylindrical shape to its best use. As explained in [25], frames serve to create a distance between the content and the surface (in this case, the column), while frameless content integrates smoothly with the surface. In order to integrate well with the column and exploit the frameless nature, frameless content is ideally suited.

\section{CONCLUSION AND FUTURE WORK}

Non-planar, digital displays have the potential to change the experience of public displays in urban spaces. Presenting content on such displays, creating an engaging user experience, and exploiting the new properties of these screens requires us to rethink the way we design content and applications. Observations of users' behavior are a necessary first step to develop guidelines to design interactive columns.

In this paper we presented the same content on a cylindrical and a planar display and observed different user behavior. We found that users move more when interacting with cylindrical displays. The sweet spot of flat displays, where users tend to position themselves, does not exist for cylindrical displays. In our study, participants spent significantly more time interacting with the flat display than with the cylindrical display. These findings can inform the design of cylindrical displays in the following way. Content for cylindrical displays should be designed for audiences in motion. Further, they are suitable to be placed in the way of users. Gestural interaction should be possible, ideally using one hand only, because users may not have their shoulders parallel to the display. As they are only semi-framed, frameless content is best suited for cylindrical displays. If a design goal is that people spend little time engaging with the display (e.g., public display in a busy area) then cylindrical screens may be more appropriate than flat ones.

We see five important limitations of this study and areas of future work. First, for this study we decided to compare cylindrical displays to flat displays that cover the same floor real estate. A follow-up study should compare both displays 
to a flat display of the size of the cylindrical display unrolled. Second, multi-user scenarios are an obvious application area for cylindrical displays. Because there is no sweet spot, multiple users can approach the display on equal grounds and do not need to take turns. For this reason users may also feel less observed as if standing in an exclusively occupied spot. Third, while our experimental setup enabled the detailed investigation of motion behavior under lab conditions, behavior in public places might differ. A field study of the prototype will be the next step in investigating the properties of cylindrical displays. Fourth, in this study we compared display formats using one specific interactive application. It would also be interesting to evaluate user behavior with different applications. Fifth, we did not investigate gaze behavior or recall of content on the displays. It would be interesting to investigate whether the increased movement when interacting with columns leads to increased recall of content.

\section{ACKNOWLEDGMENTS}

The research leading to these results has received funding from the European Union Seventh Framework Programme under grant agreements no. 215893 and 244011.

\section{REFERENCES}

1. Benko, H., Wilson, A. D., and Balakrishnan, R. 2008. Sphere: multi-touch interactions on a spherical display. In Proc. UIST '08. ACM, New York, NY, 77-86.

2. Benko, H. 2009. Beyond flat surface computing: challenges of depth-aware and curved interfaces. In Proc. ACM Multimedia. MM '09. ACM, New York, NY, 935-944.

3. Biehlig, W., Deter, C., Dube, S., Hill, B., Helling, S., Isaković, K., Klose, S., Schiewe, M. 2004. LaserCave - Some Building Blocks for immersive Screens. VR AR Statustagung, Leipzig.

4. Bly, S. A., Harrison, S. R., and Irwin, S. 1993. Media spaces: bringing people together in a video, audio, and computing environment. Commun. ACM 36, 1 (Jan. 1993), 28-46.

5. Brignull, H. and Rogers, Y. 2003. Enticing people to interact with large public displays in public spaces. In Proc. INTERACT 03, Zürich, Switzerland, 17-24.

6. Christian, A. D. and Avery, B. L. Speak Out and Annoy Someone: Experience with Intelligent Kiosks. In Proceeding of CHI 2000, The Hague, Netherlands, 2000.

7. Churchill, E., Nelson, L., Denoue, L., Girgensohn, A. 20030. The Plasma Poster Network: Posting Multimedia Content in Public Places. In Proc. INTERACT 2003, Zürich, Switzerland.

8. Churchill, E., Nelson, L., Denoue, L. 2003. Multimedia fliers: information sharing with digital community bulletin boards, Communities and technologies, Kluwer, B.V., Deventer, The Netherlands.

9. Grossman, T. and Balakrishnan, R. 2008. Collaborative interaction with volumetric displays. In Proc. CHI '08. ACM, New York, NY, 383-392.

10. Grossman, T., Wigdor, D. and Balakrishnan, R. (2004). MultiFinger Gestural Interaction with 3D Volumetric Displays. In Proc. UIST 2004. pp. 61-70.

11. Hassenzahl, M., Burmester, M., and Koller, F. 2003. AttrakDiff: A questionnaire to measure perceived hedonic and pragmatic quality. In J. Ziegler \& G. Szwillus (Eds.), Mensch \& Computer 2003. Interaktion in Bewegung, 187-196.
12. Holman, D. and Vertegaal, R. 2008. Organic user interfaces: designing computers in any way, shape, or form. Commun. ACM 51, 6 (Jun. 2008), 48-55.

13. Huang, E. M. Koster, A. and Borchers, J. 2008. Overcoming assumptions and uncovering practices: When does the public really look at public displays? In the Proc. Pervasive 2008. Sydney, Australia.

14. Jacucci, G., Morrison, A., Richard, G.T., Kleimola, J., Peltonen, P., Parisi, L., Laitinen, T. 2010. Worlds of information: designing for engagement at a public multi-touch display. In: Proc. CHI 2010, pp: 2267-2276.

15. Krueger, M., Gionfriddo, T., Hinrichsen, K. VIDEOPLACE - an artificial reality. SIGCHI Bull. 16, 4 1985, 35-40.

16. Landis, J.R., Koch, G.G. 1977. The measurement of observer agreement for categorical data. In Biometrics, 1977, Vol. 33, 159-174.

17. Lee, J. C., Hudson, S. E., and Tse, E. 2008. Foldable interactive displays. In Proc. UIST '08. ACM, New York, NY, 287290.

18. Lin, J., Chen, Y., Ko, J., Kao, H., Chen, W., Tsai, T., Hsu, S., and Hung, Y. 2009. i-m-Tube: an interactive multi-resolution tubular display. In Proc. ACM Multimedia '09. ACM, New York, 253-260.

19. Malik, S., Ranjan, A. and Balakrishnan, R. 2006. Interacting with large displays from a distance with vision-tracked multifinger gestural input. In Proc. SIGGRAPH '06. NY.

20. Manovich, L. 2001. The Language of New Media. Cambridge/Mass., MIT Press.

21. Michelis, D., Müller, J. 2010. The Audience Funnel: Observations of Gesture Based Interaction with Multiple Large Displays in a City Center. In: International Journal of HumanComputer Interaction.

22. Müller, J., Exeler, J., Buzeck, M. and Krüger, A. 2009. ReflectiveSigns: Digital Signs that Adapt to Audience Attention. In the Proc. Pervasive 2009, Nara, Japan.

23. Müller, J., Wilmsmann, D., Exeler, J., Buzeck, M., Schmidt, A., Jay, T. and Krüger, A. 2009. Display Blindness: The effect of Expectations on Attention towards Digital Signage. In the Proc. Pervasive 2009, Nara, Japan.

24. Peltonen, P., Kurvinen, E., Salovaara, A., Jacucci, G., Ilmonen, T., Evans, J., Oulasvirta, A., and Saarikko, P. 2008. It's Mine, Don't Touch!: interactions at a large multi-touch display in a city centre. In Pro. CHI '08, New York, 2008, 1285-1294.

25. Pinhanez, C. and Podlaseck, M. 2005. To frame or not to frame: The role and design of frameless displays in ubiquitous applications. In Proc. of Ubiquitous Computing, 340-357.

26. Reitberger, W., Meschtscherjakov, A., Mirlacher, T., Scherndl, T., Huber, H., and Tscheligi, M. 2009. A persuasive interactive mannequin for shop windows. In Proc. Persuasive '09, vol. 350. ACM, New York, NY, 1-8.

27. Scott, S. D., Carpendale, M. S. T., and Inkpen, K. M. 2004. Territoriality in Collaborative Tabletop Workspaces. In Proc. CSCW'04, NY, USA. ACM Press, 294-300.

28. Vogel, D. and Balakrishnan, R.: Interactive public ambient displays: Transitioning from implicit to explicit, public to personal, interaction with multiple users. In Proc. UIST '04, ACM Press, 2004, 137-14.

29. Wellner, P. 1993. Interacting with paper on the DigitalDesk. Commun. ACM 36, 7 (July 1993), 87-96. 